Revue d'histoire de l'Amérique française

RAS REVUE D.HISTOIRE DE L'AMÉRIQUE FRANÇAISE

\title{
Jean Cabot et la découverte de l'Amérique du Nord
}

\section{Lucien Campeau}

Volume 19, numéro 3, décembre 1965

URI : https://id.erudit.org/iderudit/302491ar

DOI : https://doi.org/10.7202/302491ar

Aller au sommaire du numéro

Éditeur(s)

Institut d'histoire de l'Amérique française

ISSN

0035-2357 (imprimé)

1492-1383 (numérique)

Découvrir la revue

Citer cet article

Campeau, L. (1965). Jean Cabot et la découverte de l’Amérique du Nord. Revue d'histoire de l'Amérique française, 19(3), 384-413.

https://doi.org/10.7202/302491ar d'utilisation que vous pouvez consulter en ligne.

https://apropos.erudit.org/fr/usagers/politique-dutilisation/ 


\section{JEAN CABOT \\ ET LA DÉCOUVERTE DE L'AMÉRIQUE DU NORD *}

Le retour de Christophe Colomb en Espagne, en 1493, a soulevé dans toute l'Europe un enthousiasme compréhensible pour la découverte des terres inconnues. Il y eut foule d'aspirants-découvreurs, désireux de partager la gloire du Génois et encore bien plus les avantages financiers de son aventure. Il y eut des découvreurs sérieux et il y en eut aussi qui n'étaient que des intrigants et des filous. Les géographes étaient alors à l'affût de toute nouvelle information sur les parties inconnues de notre sphère. Il en arriva en fait de toutes parts et on les inscrivit sur les cartes. Les débuts de la cartographie américaine en témoignent. Dans l'ouest de l'Atlantique émergea la fameuse côte est-ouest de La Cosa ${ }^{1}$, puis la côte nord-sud qui a quelquefois pour nom Cuba ou Bimini ${ }^{2}$, puis le Groenland-Labrador, avec la terre de Cortereal ${ }^{3}$, une île de Labrador sur la carte Oliveriana-Pesaro ${ }^{4}$, l'île des Sept-Cités, l'île de Brasil, l'île de Saint-Brandan et d'autres encore. Les cartographes n'inventaient

* Travail présenté à la réunion générale de l'Institut d'Histoire de l'Amérique française, le 1er mai 1965.

1 Voir une excellente reproduction de cette carte dans Mémoires de la Société Royale du Canada (MSRC), II, avant la p. 269.

2 Cette terre apparaît le plus souvent sans nom sur les plus anciennes cartes de l'Amérique du nord, sur celles de Cantino, Caverio, Waldseemüller, etc. Le dessin est probablement d'origine portugaise (B. Hoffman, Cabot to Cartier, Sources for a Historical Ethnography of Northeastern North America, 1497-1550 (Toronto, [ 1961 ]), fig. 4 et suivantes).

3 Ces deux dernières terres apparaissent d'abord sur des cartes portugaises. Les monuments de la cartographie portugaise ont été richement édités par Armando Cortesao et Avellino Teixera da Mota, Portugalliae Monumenta cartographica, Comemorações do $V$ Centenario da Morte do Infante D. Enrique, Lisboa, 1960. Le premier des cinq volumes contient les plus anciennes cartes qui concernent l'Amérique du Nord.

4 Voir une reproduction dans Ymago Mundi, VII (1950): 82. 
pas ces détails; ils rapportaient ce qu'on leur affirmait. Mais il leur arrivait aussi, par ignorance ou par préjugé, de créer des fantômes qui les hantaient ensuite quelque temps. Surtout au début de la cartographie américaine, ils n'avaient pas les moyens de vérifier et de trier ces témoignages.

Ils ne manquaient cependant pas d'esprit critique; on le vit bientôt. La côte de La Cosa, représentation espagnole, ne fit qu'un séjour très court dans la cartographie. L'île de Labrador n'y fut guère plus longtemps. La terre de Cuba-Bimini résista davantage, puis elle s'engloutit dans l'Atlantique à l'apparition de la Floride. D'autres îles flottèrent plus ou moins longtemps, errant çà et là, puis elles sombrèrent à leur tour. De ce qui sera par la suite l'Amérique du Nord, il ne restait plus guère, vers 1520, que la terre de Cortereal, qui avait pris racine en 1502 dans le nord, et la Floride, à proximité de Cuba. Presque tout le reste avait été reconnu faux. La science moderne devrait voir dans ce fait un avertissement. Ce qui a été pour la cartographie américaine des erreurs de jeunesse et d'ignorance, rapidement corrigées, ne doit pas être tenu par notre critique sourcilleuse pour des témoignages authentiques et irrécusables. Les déchets de la critique ancienne ne sauraient, en règle générale au moins, devenir pour nous les matériaux solides de l'histoire.

Une chose me paraît démontrable, sinon évidente à première vue. De tous les dessins que l'on met dans l'atlantique-nord durant les premières années du seizième siècle, la côte de Cortereal est le seul qui ait résisté à la critique des savants de ce temps et ait formé le noyau le plus ancien d'une représentation de l'Amérique du Nord. Or ce dessin est sans conteste inspiré par la découverte de Gaspar Cortereal, en 1501, et il ne doit absolument rien à Jean Cabot. C'est une tâche vraiment désespérée que de vouloir relever dans l'évolution ultérieure de ce littoral des traces du passage du Vénitien, ainsi que l'a fait Ganong avec la plus prodigue des éruditions ${ }^{5}$. Les cartes portugaises

* La remarquable étude de W. F. Ganong, publiée dans les MSRC à partir de 1929, a été réunie en un seul volume et récemment rééditée par Theodore E. Layng (W. F. Ganong, Crucial Maps in the early Cartography and Place-Nomenclature of the Atlantic Coast of Canada (Toronto, [1964]). 
trahissent bien quelque fréquentation française du nouveau continent - par exemple, le cap Breton - mais absolument rien d'un passage de Cabot. Je me laisserais entraîner trop loin de mon sujet, si j'entrais dans une démonstration de ce point. C'est pourquoi je ne fais ici que le mentionner, me limitant aux documents qui concernent les voyages de Jean Cabot. Je ne m'arrêterai pas ici aux racontars de Sébastien, son fils, dont j'ai suffisamment parlé autrefois ${ }^{6}$.

Je discuterai deux versions de la découverte de Jean Cabot. La première sera celle que je prétends tirer de la lettre de Raimondo di Soncino au duc Lodovico-Maria Sforza de Milan, écrite de Londres, le 18 décembre $1497^{7}$. La deuxième semble ressortir de la lettre d'un certain John Day à un "Almirante mayor" de Castille, sans lieu de provenance, sans date et sans mention explicite du découvreur ${ }^{8}$. Le reste de la documentation sera groupé autour de l'une ou de l'autre de ces pièces, selon les exigences de la critique. Car ces autres documents ne sont en eux-mêmes ni assez précis ni assez complets pour former à eux seuls une preuve.

La première version est donc celle qui me paraît ressortir de la lettre de Soncino. Ce document daté et signé par un personnage responsable, qui contient le nom du découvreur et se rapporte sans aucun doute possible à Jean Cabot, me paraît capital. L'original, entièrement écrit de la main du signataire, est conservé à l'Archivio de Milan, Fondo Sforzesco, Potenze estere Inghilterra, cartello 567. La lettre comporte deux feuillets de $0,293 \times 0,211 \mathrm{~m}$. Le texte occupe les deux côtés du premier et le recto du second, tandis que la suscription est au verso de ce dernier. Le texte de Biggar est assez bon, ne comportant que des fautes minimes, surtout sur le mot rotundia, qui est écrit

6 "Les Cabot et l'Amérique", Revue d'Histoire de l'Amérique francaise, XIV (1960): 317-352.

7 Texte italien et traduction anglaise dans H. P. Biggar, Les Précurseurs de Jacques Cartier, 1497-15\$4 (Ottawa, 1913), 17-21.

8 Texte espagnol publié par L. A. Vigneras, "New Light on the 1497 Cabot Voyage", Hispanic American Historical Review, XXXVI (1956): 503-509; du même auteur, "The Cape Breton Landfall: 1494 or 1497", The Canadian Historical Review, XXXVIII (1957): 219-228. 
correctement rotundità dans le manuscrit. La suscription est aussi notablement altérée chez Biggar ${ }^{9}$. Cette lettre, écrite de Londres, fait partie d'une série d'autres également reçues d'Angleterre.

Soncino ne parle pas seulement d'après les rumeurs, dans la lettre que nous étudions. En décembre, il est déjà l'ami de Jean Cabot, qui le créerait archevêque des terres nouvelles, si le Milanais avait le goût des aventures. Il a causé longuement avec le découvreur, examinant la mappemonde de ce dernier et le globe fabriqué par lui. Cabot lui a montré le chemin suivi pas son navire et lui a raconté de vive voix son voyage, lui faisant aussi part de ses projets. A moins de tenir Soncino pour un menteur, il faut croire que Cabot lui a raconté tout cela. Et si on lui refuse foi, c'est le meilleur témoin de la découverte qu'on se trouve à récuser.

Mon interprétation de la lettre de Soncino a fait scandale ${ }^{10}$. On voudra bien d'abord ne pas se laisser embrouiller par les détails. Le fondement essentiel de ma thèse est cette description: "Ce messer Jean possède la description du monde sur une carte et aussi sur une sphère solide que lui-même a fabriquée. Il montre où il est arrivé; et voyageant vers le levant, il a dépassé de beaucoup le pays du Tanaïs... Mais messer Jean a dans l'esprit de plus grands projets encore. Il a l'intention, partant de ce lieu après l'avoir occupé, de s'en aller toujours côtoyant le rivage vers le levant, tant qu'il ne sera pas vis-à-vis d'une île appelée par lui Cipango, située dans la région équinoxiale, où il croit que prennent origine toutes les épices du monde et aussi les pierres précieuses" "11. Ce que je maintiens est ceci: un voyage

9 Elle se lit comme suit "Ill[ustrissi]mo ac Ex[cellentissi]mo principi / et d[omi]no meo colen[dissi]mo / Duci Medi[ol]ani / ray[mondus]".

10 Cf. supra, n. 6.

11 "Esso messer Zoanne ha la descriptione del mundo in una carta et anche in una sphera solida che lui ha fatto, et demonstra dove è capitato, et andando verso el levante ha passato asai el paese del Tanais... Ma messer Zoanne ha posto l'animo ad magior cosa, per che pensa da quello loco occupato andarsene sempre a riva riva piu verso el levante, tanto ch'el sia al opposito de un isola da lui chiamata Cipango, posta in la regione equinoctiale, dove crede che nascano tutte le speciarie del mundo et anche lee gioie." 
qui part de l'Angleterre, passant par le pays du Tanaïs et devant se terminer vis-à-vis de Cipango, ne peut se faire qu'en direction de l'est.

On connaît la manière dont on se représentait le monde à la fin du quinzième siècle. C'était en principe celle de l'antiquité gréco-romaine, dont le plus grand nom, à ce propos, était Ptolémée. Cette conception était essentiellement une représentation de Méditerranéens, qui se voyaient eux-mêmes au milieu du monde, dans la situation la plus favorable au développement humain. La planète est une sphère, dont l'océan et la terre ferme se partagent la surface. On ignore quelle est la proportion de mer et de terre sur la planète. Mais il existe un système de conceptions traditionnelles sur la partie qui est habitée.

Encore en $1490^{12}$, la terre des cosmographes, c'est-à-dire celle où vivent les divers peuples, est représentée comme un plateau plus ou moins circulaire, ou plutôt comme une calote posée sur la sphère et divisée en trois parties: l'Asie, d'une part, l'Europe et l'Afrique, de l'autre. L'Asie est la moitié orientale de ce cercle; l'Europe est le quart nord-ouest; l'Afrique est le quart sud-ouest. La Méditerranée sépare ces deux dernières, occupant à peu près la moitié du diamètre ouest-est. Mais l'Europe est séparée de l'Asie surtout par le fleuve Tanaïs. Voici comment, en 1507, Waldseemüller décrivait les bornes de l'Europe: "Europa ab occidente mari Athlantico, a septentrione Britannico, ab oriente Thannai, Meotide palude et Ponto, a meridie mari Mediterraneo clauditur". Et il s'exprime encore de même à propos de l'Asie: "Asia ... ab Europa Thanai fluvio... secernitur" ${ }^{13}$. Ainsi, le Tanaïs est, dans la géographie ancienne, plus qu'un fleuve réputé; il est la frontière traditionnelle et classique entre l'Europe et l'Asie. Et le pays du Tanaïs ne peut être, pour un navigateur qui en approche par l'océan,

12 Edmond Buron, Ymago Mundi de Pierre d'Ailly, (3 vol., Paris, [1930]). On trouvera dans cette œuvre plusieurs reproductions de ce genre de cartes (pl. XXXII et XXXIII). Plus importante que les autres est celle qui a été faite entre 1482 et 1488 (pl. XXIV) et appartient toujours à ce type. Elle est contemporaine de Cabot.

13 Martin Waldseemüller, Cosmographiae Introductio..., (Saint-Dié, 1507), sans pagination. 
que la contrée montagneuse où ce fleuve prend sa source, au septentrion de la terre habitée ${ }^{14}$. Car on ne connaît que ce Tanaïs. Il n'y en a pas d'autres sur ce continent circulaire et tripartite, que l'océan entoure de toutes parts sur bon nombre de cartes.

Cette conception circulaire de la terre habitée est celle de Jean Cabot. On en a la preuve dans l'un de ses raisonnements, rapporté par Soncino. Cabot s'était autrefois rendu jusqu'à La Mecque, où il avait vu arriver les caravanes apportant les épices et les joyaux de l'orient. Il s'était informé de l'origine de ces marchandises. Les caravaniers lui avaient répondu qu'ils les avaient eux-mêmes de caravanes venues de plus loin, qui les achetaient à d'autres de régions plus lointaines encore, et ainsi de suite. Soncino poursuit: "Et il fait ce raisonnement que, si les orientaux affirment aux méridionaux que ces choses viennent d'un pays éloigné d'eux, et ainsi de main en main, présupposée la rotondité de la terre, il est nécessaire que les derniers les prennent au septentrion, vers l'occident" ${ }^{15}$. La rotundità de la terre ne peut signifier ici en aucune façon la sphéricité du globe. Car elle est la forme du continent qui conditionne le trajet des caravanes, lesquelles, cela va de soi, ne voyagent que par terre. Les méridionaux sont les habitants de La Mecque, cette ville se trouvant dans la moitié sud de l'Asie, à quelque dix degrés plus bas que le diamètre qui réunit l'occident à l'orient. Les orientaux sont appelés ainsi par rapport à La Mecque. Si l'on refait à l'inverse la route des caravanes, en se dirigeant vers

14 S. E. Dawson ("The Voyages of the Cabots", MSRC (1897), II: 232-240) s'est acharné à identifier le "pays du Tanaîs" avec une contróe déterminée que les anciens auraient appelée de ce nom. L'utilité qu'il eût pu tirer de là pour prouver sa théorie d'un atterrage de Cabot au cap Breton est fort problématique. Le malheur a voulu, au surplus, qu'il ait cherché une preuve dans une figure de Pierre d'Ailly, qu'il a prise pour une carte géographique et qui n'en est pas une (ibid., 237). C'est un schéma illustrant les divers climats et leur contenu. Le Tanaîs y est inscrit en travers, sur deux climats. Ce n'est pas une raison pour en faire un pays. L'Indus et le Nil se présentent de la même façon et personne n'a jamais prétendu pour cela qu'ils étaient des pays.

15 "Et fa questo argumento, che se li orientali affermanno a li meridionali che queste cose venghono lontano da loro, et cosi de mano in mano, presupposta la rotundità de la terra, é necessario che li ultimi le tolliano al septentrione verso l'occidente." 
l'est, on atteint la circonférence de la terre et l'on est forcé, pour continuer sa route, de remonter vers le nord en suivant cette circonférence. Poursuivant toujours plus loin, on traverse le diamètre terrestre à l'orient et la route s'incurve désormais vers l'ouest, à cause de la courbure du cercle. C'est ainsi que, selon Cabot, l'origine des épices et des joyaux doit logiquement se trouver au nord vers l'occident (fig. I). En d'autres mots, la route des caravanes, suivie à rebours, vient à la rencontre d'un navire qui, venant d'Angleterre en contournant le nord du continent, suivrait le rivage de l'Asie en descendant vers le sud-est.

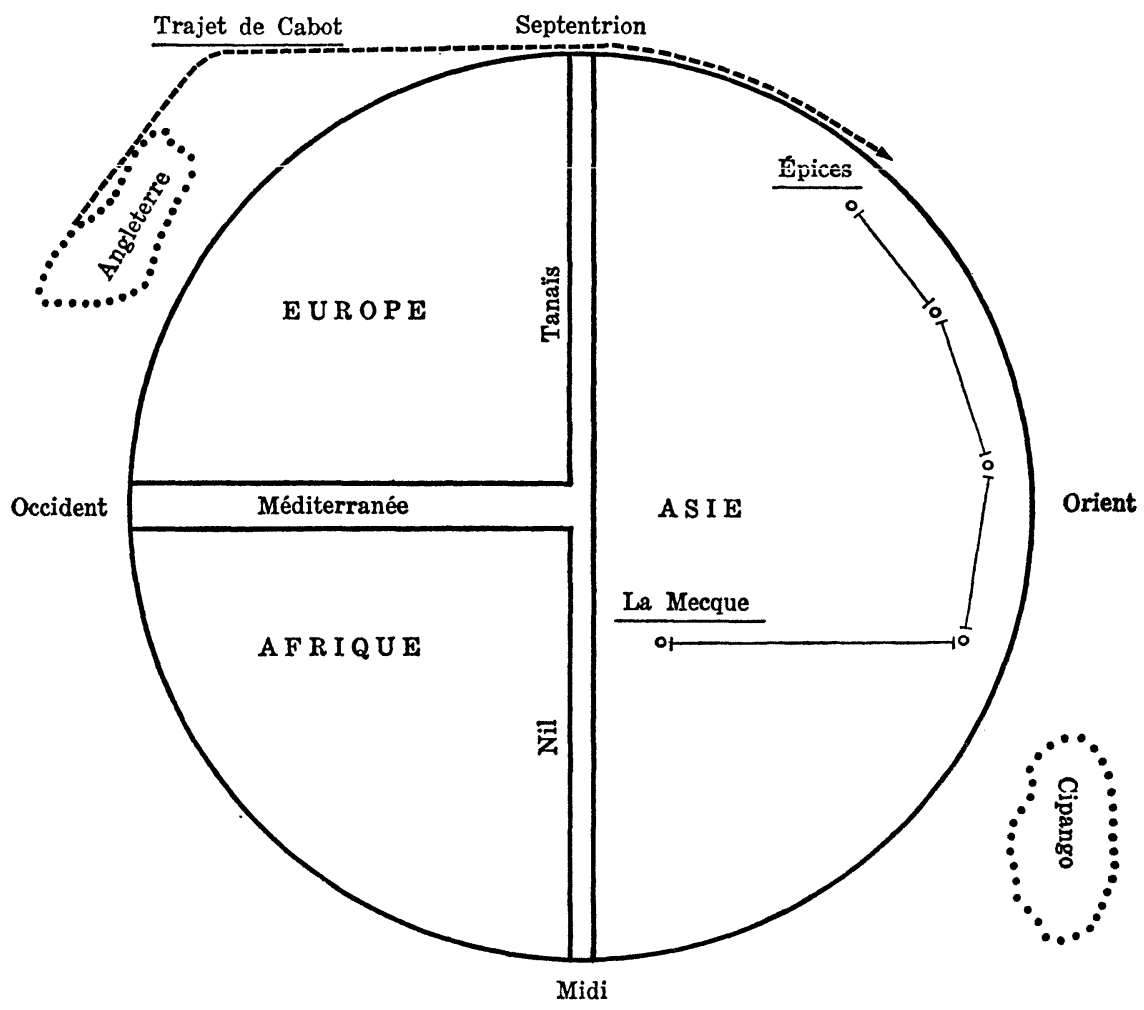

Fig. 1 - Schéma indiquant les divisions de la terre habitée, au quinzième siècle, et illustrant les conceptions de Jean Cabot. 
L'idée de contourner la terre habitée par le nord pour aller d'occident en orient était fort plausible au temps de Jean Cabot. Le cercle des terres connues, l'orbis terrarum des anciens, était à peu près compris entre le $63^{\circ}$ degré de latitude nord et le $16^{\circ}$ degré de latitude sud ${ }^{16}$. On imaginait d'ordinaire ce cercle comme entièrement entouré par l'océan. Les Portugais cherchaient à le contourner par le sud. Avant 1490, leurs explorations leur avaient fait trouver la route vers l'orient près du $35^{e}$ degré de latitude australe ${ }^{17}$. Du même coup, on avait appris que la navigation de l'Asie par le sud serait plus longue que prévue. D'autre part, Christophe Colomb s'était convaincu, en lisant les anciens cosmographes et surtout Pierre d'Ailly, que les deux extrémités du diamètre ouest-est du continent, les îles Canaries dans l'Atlantique et l'extrémité mystérieuse de l'Inde à l'opposé, étaient peu éloignées l'une de l'autre par une navigation directe à travers l'océan. Il suffirait, pensait-il, de quelques jours de navigation par vent favorable ${ }^{18}$. L'expérience lui apprit que le chemin était plus long, puisqu'il mit environ 65 jours à atteindre la première île de l'Asie, selon ce qu'il croyait. On ne considère pas assez qu'il restait une troisième voie, c'est-à-dire une circumnavigation par le nord de la terre. Colomb lui-même s'y était arrêté et s'était convaincu de sa possibilité. Car la lecture de Pline lui apprenait que la mer Septentrionale était libre de glaces ${ }^{19}$.

Ici, le témoignage de Sébastien Cabot va nous servir à quelque chose. Après le voyage de Chancelor au nord de la Russie, en 1553-1554, notre Sébastien, encore une fois avant sa mort, n'a pu résister à la tentation de se faire passer auprès de ses amis italiens pour le découvreur de la route de la Chine. Il s'attribue donc une expédition qu'il aurait faite en 1556-1557 jusqu'à l'Ob, fleuve russe qui coule à l'est de l'Oural et de la

16 Buron, Ymago Mundi, $3: 559$.

17 Barthélemi Diaz avait dépassé le cap de Bonne-Espérance et reconnu l'orientation nord-est de la côte africaine au-delà de ce point, en 1488.

18 Buron, Ymago Mundi, $3: 661$.

10 Colomb faisait cette annotation en marge du texte de Pline: "Auctor oceanum septentrionalem non est concretum neque innavigabile; orientem nobis incognitum veteres tamen navigatum produnt" (Buron, Ymago Mundi, $3: 743$. 
Nouvelle-Zemble ${ }^{20}$ Les historiens n'ont pas pris ces vantardises au sérieux, cette fois. Mais, dans une préface à son récit, Sébastien raconte que l'idée d'un passage vers la Chine par le nord avait toujours été chèrement caressée par son père et par luimême. Il confie que ce dessein était toujours demeuré secret dans son cœur, même lorsqu'il était en Espagne, car il en réservait les avantages à l'Angleterre. La fondation de la compagnie de Moscovie - qui a été provoquée en fait par la découverte de Chancelor - lui a enfin donné le moyen de réaliser son rêve. Jean et Sébastien Cabot fondaient leurs espoirs sur certains récits empruntés par Pline à Cornelius Nepos et sur un épisode du temps d'Othon I, où des marins venus de l'Inde avaient échoué sur la côte septentrionale de l'Europe. Tout cela pour montrer qu'il ne faut pas se surprendre de ce que Jean Cabot a raconté à Soncino.

Ainsi, d'après Soncino, Jean Cabot a défini sa trajectoire avec une netteté impérative et irrécusable. L'Angleterre, qui se trouvait déjà dans la mer Septentrionale, au-dessus même des climats théoriquement habitables, selon l'opinion d'alors, était un point de départ naturel pour une telle expédition. Le Tanaïs était le fleuve renommé que j'ai dit plus haut, un point de repère qui ne donnait lieu à aucune ambiguïté. Quant à Cipango, moins notoire, elle était quand même bien connue des géographes. Cette île, plus étendue du nord au sud que de l'est à l'ouest, était indiquée sur les cartes à proximité de la côte orientale de l'Asie, en grande partie au-dessous du tropique du Cancer. Marco Polo avait naguère signalé son existence et on l'identifiera plus tard avec le Japon. Un voyage maritime qui part de l'Angleterre, dépasse par le nord la frontière séparant l'Europe de l'Asie et s'achemine vers Cipango ou le Japon ne peut pas ne pas se diriger vers l'est. J'en suis désolé pour les tenants d'une découverte américaine par Jean Cabot en 1497. Mais l'explorateur a luimême, selon le seul témoin qui soit explicite sur le sujet, affirmé avoir pris une direction opposée.

20 "Navigatione di Sebastiano Cabota", dans G. B. Ramusio, Secondo volume delle navigationi ..., (Venetia, 1574), [211]-219. 
S'il avait voulu trouver Cipango par l'ouest, il aurait fallu prendre la route du sud-ouest, au lieu de commencer par monter au nord. Tout le monde voyant Cipango au sud-est de l'Asie, on ne pouvait, dans cette hypothèse, $\mathrm{y}$ aller qu'en traversant la trajectoire de Colomb. En effet, après 1492, on situait cette île à peu près sur le site actuel du Vénézuela. Le Tanaïs se fût trouvé alors dans une direction absolument divergente et sur une longitude beaucoup plus occidentale que Cipango. Jean Cabot a donc bien parlé d'un voyage par le nord de l'Europe et de l'Asie.

La direction du voyage de Cabot n'est pas seulement déduite des points qui la définissent géométriquement. Elle est explicitement donnée par la lettre de Soncino, à trois reprises: “... s'étant élevé vers le septentrion, il commença à naviguer à la partie orientale ..."; "... et allant vers le levant, il a dépassé de beaucoup le pays du Tanaïs”; “... il pense s'en aller de ce lieu, après l'avoir occupé, côtoyant toujours le rivage vers le levant, jusqu'à ce qu'il soit vis-à-vis d'une île appelée par lui Cipango". J'ai un peu honte d'expliquer ces termes, surtout après ce que je viens de dire sur le sujet du Tanaïs. Mais puisqu'on me fait des objections sur le sens des mots orient et levant, il ne sera peutêtre pas inutile que je m'y arrête.

Orient et levant ont évidemment un rapport avec le côté où le soleil se lève. Géographiquement, l'orient est le point de roncontre entre l'horizon et son diamètre perpendiculaire à l'étoile polaire, du côté où le soleil apparaît. Chaque lieu a ainsi son orient, mais les astrologues du Moyen Age parlaient aussi de l'orient de la terre habitée, le point de rencontre du diamètre de cette terre avec sa circonférence, du côté du soleil levant. Cet orient était dans l'Inde mystérieuse, à l'opposé des îles Canaries.

Telle est la signification d'orient et de levant. Et toute autre, s'il s'en trouve, ne peut qu'être dérivée de celle-là. On m'objecte que, dans le texte de Soncino, ces deux mots sont des noms de pays, vers lesquels on se dirige en mettant le cap à l'ouest. Vu le sens naturel des vocables, c'est à mes objecteurs de prouver la validité de leur objection. En attendant qu'on me fournisse cette preuve, je dirai ceci. Il faut oublier que nous sommes Amé- 
ricains et du vingtième siècle. Pour un Américain d'aujourd'hui, il est facile de considérer l'Asie comme située à l'ouest, où seulement un océan nous en sépare, tandis que de l'autre côté se trouvent un océan et un continent entre elle et nous. Mais d'où vient à l'Américain moderne ce mot East, par lequel il désigne parfois l'Asie en un sens contraire à l'étymologie ? Il vient d'Europe, où l'Asie apparaît effectivement à l'est, et non à l'ouest. En 1497, il n'y avait pas d'Américains parlant les langues occidentales. Les Européens du temps, s'ils faisaient d'orient et de levant des noms de pays, ne pouvaient que les situer à l'est. D'abord, parce qu'il n'était pas entré dans l'usage de voir l'Asie comme accessible par l'ouest. Et puis, parce qu'en fait ce continent se trouvait tout près d'eux à l'est. C'est ce que prouvent les expressions Proche-Orient, Moyen-Orient, Extrême-Orient, Near-East, Middle-East, Far-East, employées en ces derniers siècles. Toutes ces parties de l'Asie ne peuvent être désignées de la sorte que si elles sont vues à l'est. Autrement, ce serait le Far-East qui serait proche et le Near-East qui serait loin. Aussi, pour un Milanais de 1497, employer, sans aucune explication, les mots orient et levant dans le sens d'occident, c'eût été tromper son lecteur.

D'ailleurs, Soncino ne parle pas de pays, mais bien de directions et d'orientations. Il ne dit pas "verso l'Oriente", mais "a le parte orientale". Il y a ici difficulté grammaticale: l'article est au pluriel, mais le nom et l'adjectif sont au singulier. Faut-il traduire "vers les parties orientales" ou bien "du côté oriental" ? J'abandonne la réponse au lecteur. Mais lorsque Soncino écrit qu'en allant "verso il levante", Cabot a dépassé le Tanaïs et se trouve en Asie, le sens est inévitable: on navigue vers l'est. Car si Cabot dépassait le Tanaïs en naviguant vers l'ouest, il se trouverait de nouveau en Europe. Plus loin et une fois parvenu en Asie, il va naviguer encore plus "verso il levante". Comme il se trouve déjà dans le Levant, il faut bien, encore une fois, que "verso il levante" signifie "vers l'est".

On peut me faire une difficulté sur cette phrase: "commenciò ad navigare a le parte orientale, lassandosi, fra qualche giorni, 
la tramontana ad mano drita". Il y a deux fonctions possibles pour ad mano drita. Ou bien il est complément de lassando: laissant l'étoile polaire à sa main droite. Ou bien il explique le sens de $a$ le parte orientale: c'est-à-dire à main droite. Le premier sens contredit le contexte; le second s'y conforme. Est-ce que j'ai tort de dirimer une ambiguïté en invoquant le contexte? On ne peut pas davantage utiliser la première interprétation contre moi que je ne pourrais utiliser la seconde contre mes antagonistes. Mon interprétation me paraît se justifier d'autant mieux qu'on a le verbe impersonnel lassandosi, et non lassando, qui aurait pour sujet Jean Cabot. Ainsi, faut-il traduire, je pense: "à la partie orientale, [c'est-à-dire] à main droite, quand, après quelques jours, on laisse l'étoile polaire".

Jean Cabot a donc déclaré qu'il avait découvert l'Asie en contournant la terre par le nord de l'Europe et de l'Asie. On ne peut évidemment croire qu'il a réellement accompli cet exploit. La raison de ce mensonge était-elle de se procurer les fonds qui lui manquaient pour exécuter un projet semblable ? Je crois la chose probable. C'est peut-être là qu'il faudrait chercher la cause du silence qui va envelopper son nom durant le prochain demi-siècle. Si, en 1498 , il a conduit par là la flottille qui lui fut alors confiée, il s'en allait ou vers une tragédie ou au moins vers un échec. L'imposture ne pouvait non plus manquer d'éclater au grand jour.

Les documents contemporains n'infirment pas le témoignage de Soncino. D'après Lorenzo Pasqualigo ${ }^{21}$, Cabot était parti chercher des îles nouvelles. L'explorateur aurait, en fait, touché "la terre ferme qui est le pays du grand Khan" à $\mathbf{7 0 0}$ lieues de l'Angleterre. Il l'aurait aussi côtoyée l'espace de 300 lieues. Je fais remarquer que, dans cette question, chacun choisit les chiffres qui vont le mieux à sa thèse. Deux documents, l'extrait de dépêche dont je parlerai dans un moment et la lettre de Pedro de Ayala, donnent comme distance 400 lieues. On préfère d'ordinaire les 700 lieues, parce qu'elles rapprochent davantage de l'Amérique. N'était cette préoccu-

21 Biggar, Les Précurseurs, 13-15. 
pation, on pourrait peut-être concilier les deux groupes de témoignages en disant que les trois cents lieues faites le long du littoral sont à prendre sur les sept cents. Ce qui ferait encore une distance de 400 lieues entre l'Angleterre et le commencement du pays du grand Khan. En tout cas, Pasqualigo ne dit pas si l'Asie a été atteinte à l'ouest ou à l'est de l'Angleterre. En sorte qu'on ne sait pas si le rapport de Soncino se trouve ici confirmé ou contredit.

L'extrait de dépêche daté du 24 août $1497^{22}$ et attribué à Soncino par Biggar n'a pas ce Milanais comme auteur. L'agent du duc de Milan débarquait à Douvres à cette même date et une lettre écrite par lui ce jour même ne dit pas un mot de Cabot. L'original de la dépêche n'a pas été conservé. Ce qui en est resté est un résumé de la main d'un secrétaire et c'est le texte publié par Biggar. Cette note attribue à Cabot la découverte de deux îles, très grandes et fertiles, "et etiam" de celle des Sept-Cités, à 400 lieues de l'Angleterre, par ou sur le chemin de l'ouest: "per lo camino de ponente". De quel chemin de l'ouest s'agit-il ? De celui qu'on a fait en partant de l'Angleterre ou bien de celui qu'on a fait en revenant? Il se pourrait que cette deuxième interprétation soit la bonne, puisque l'expression se rapporte plutôt à l'île des Sept-Cités, découverte en dernier lieu. Quelle part d'interprétation le secrétaire a-t-il mis dans ce résumé ? Il ne me semble pas que cette source, ni par sa clarté, ni par son autorité, soutienne la comparaison avec la lettre de Soncino.

Enfin, on possède encore la lettre de Pedro de Ayala aux souverains d'Espagne, du 25 juillet $1498{ }^{23}$. En gros, l'Espagnol soupçonne Cabot d'avoir atteint la zone qui a été réservée à son pays par le traité de Tordesillas. Ses raisons ne sont pas tirées du récit de l'explorateur, mais des constatations qui suivent: "Vu la route qu'ils prennent et la longueur du chemin, je trouve qu'ils ont découvert ou cherchent ce que Vos Altesses possèdent, parce que c'est au cap qui est échu à Vos Altesses

22 Ibid., 15-16.

23 Ibid., 27-29. 
par l'accord fait avec le Portugal 24." Le mot cabo a une multitude de sens en espagnol. Comme on ne connaît aucun cap sur lequel on ait alors fixé la démarcation de Tordesillas, Ayala veut sans doute dire que la seconde expédition de Cabot, laquelle reprend le chemin de la première, se dirige vers la ligne de Tordesillas, donc vers l'ouest. L'expédition quitte un port de la côte ouest de l'Angleterre et la distance donnée, 400 lieues, rapproche considérablement, sinon entièrement, de cette ligne ${ }^{25}$. Remarquons toutefois que ces déductions sont celles d'Ayala et non le résultat d'informations faisant autorité. Le roi Henri VII, à qui l'ambassadeur en a parlé, les a niées. L'Espagnol a en main une carte de Cabot, mais il la tient pour fausse, parce qu'elle ne confirme pas ses soupçons. Un tel témoin ne peut évidemment pas nous renseigner sur la route qu'a suivie le Vénitien.

Voilà pour les témoins assurés de l'événement. Ce qu'on en peut tirer en faveur d'une découverte de l'Amérique du Nord par Jean Cabot est fort maigre. Il y a bien un "per lo camino de ponente" qu'on pourrait, peut-être, mettre à l'actif du découvreur. Mais il y a d'autre part le formidable témoignage de Soncino. Le moins qu'on puisse dire est que la thèse d'une découverte américaine n'est pas prouvée par là.

Reste maintenant à examiner une autre série de documents que les conjectures des historiens rattachent à cette découverte de Jean Cabot. Ce sont la lettre de John Day au grand Amiral de Castille et la carte de Juan de La Cosa.

24 "Yo, vista la derrota que llevan y la cantidad del camino, hallo que es lo que han hallado o bu[s]can lo que Vuestras Altezas poseen, porque est al cabo que a Vuestras Altezas cupo por la convenencia con Portugal" (Biggar, Les Précurseurs, 27).

${ }^{25} \mathrm{La}$ ligne établie par le traité de Tordesillas, du 7 juin 1494, passait à peu près au 47 e degré de longitude ouest de Greenwich. Elle se trouvait ainsi à environ 534 lieues à l'ouest de Bristol. 
La lettre a été découverte, en 1956, aux archives de Simancas ${ }^{26}$. Elle est écrite en espagnol et signée par un nommé Johan Day, dont on ne connaît que le nom et qui a l'air d'un marchand anglais récemment débarqué en Espagne. Elle est adressée à un Almirante mayor, pour lequel le scribe a tous les égards dus à un grand seigneur. Le grand Amiral de Castille était Fadrique Enriquez, mais certains voudraient identifier le destinataire avec Christophe Colomb. La lettre n'a pas de date. Le découvreur dont il est question n'est pas nommé. M. L. A. Vigneras a fort bien exposé les raisons qui paraissent mettre ce document en relation avec l'expédition de Jean Cabot, en 1497: "Here are some of the details given by John Day which check with information derived from the other sources: the voyage lasted three months, from May to August; the new land was identified as the Island of the Seven Cities; Cabot took possession of it in the name od the King of England; he saw no human beings but found signs that the land was inhabited; he noticed an amazing quantity of codfish; upon his return, he was granted an annual pension of twenty pounds; and a much larger expedition was planned for the following year ${ }^{27}$."

Il y a cependant quelques divergences. Les distances ne sont pas les mêmes: 1800 milles pour le point le plus rapproché d'Irlande contre 700 lieues, ou 400 qui sont mieux attestées dans l'autre série de documents. Selon la lettre de Day, le voyage aurait duré 35 jours pour l'aller; l'exploration aurait pris environ un mois et le retour aux côtes de l'Europe se serait fait en 15 jours, plus quelques jours pour revenir à Bristol. Comme il est certain que Jean Cabot était revenu de son voyage plusieurs

26 Texte espagnol publié par L. A. Vigneras, "New Light on the 1497 Cabot Voyage", Hispanic American Historical Review, XXXVI (1956) : 503-509; traduction anglaise après l'article cité à la note suivante. L'antiquité de ce document est mise en doute par quelques partisans de la découverte de l'Amérique du Nord par Cabot. Mais ceux-là mêmes admettent que le papier est ancien. L'écriture paraît pourtant bien d'époque, à cause de son caractère fortement gothique. Jusqu'à preuve du contraire, je tiens cette pièce pour un document du début du seizième siècle.

27 L. A. Vigneras, "The Cape Breton Landfall: 1494 or 1497", The Canadian Historical Review, XXXVIII (1957): 221-222. 
jours avant le 10 août $1497^{28}$, le départ se trouve reporté, non pas à la fin de mai, mais au milieu de ce mois au plus tard. Une autre difficulté vient de ce que la lettre mentionne une découverte antérieure des marchands de Bristol. Mais il semble certain qu'il n'y en eut aucune avant $1503^{29}$. Il y a, évidemment, une opposition diamétrale avec le récit de Soncino concernant la direction des voyages. Mais un autre fait me rend cette lettre suspecte. C'est le ton hypocrite et vénal du personnage, qui, multipliant les professions de loyauté au roi d'Angleterre, livre à un haut officier de la reine de Castille des informations de la plus grande importance, que Henri VII ne serait certainement pas heureux de voir divulguées en Espagne. Car cette terre neuve, qui fait pendant à l'Europe, à 1800 milles à l'ouest de la côte d'Irlande, ne peut pas manquer de se trouver dans l'hémisphère colonial de l'Espagne. La distance et la direction ne pourraient pas être mieux calculées pour soulever la jalousie des Castilians. Et le grand Amiral, que ces matières concernent au premier chef, sera incapable de garder secrète une telle information.

28 Biggar, Les Précurseurs, 12.

29 Le 19 mars 1501, Henri VII autorisait un groupe de marchands anglais de Bristol et quelques navigateurs portugais à noliser à leurs frais le nombre voulu de navires, pour aller "ad omnes partes, regiones et fines maris Orientalis, Occidentalis, Australis, Borialis et Septentrionalis ... ad inveniendum, recuperandum, discooperiendum et investigandum insulas, patrias, regiones sive provincias quascumque gentilium et infidelium in quacumque mundi parte positas, quæ Christianis omnibus ante hæc tempora fuerunt et inpræsenciarum sunt incognite...". Si les Anglais, à ce moment, avaient eu connaissance d'une nouvelle terre, déjà découverte, la chose la plus logique eût été d'adresser les explorateurs à ce pays nouveau, pour en poursuivre l'exploration et en rapporter des informations plus complètes. Même en supposant, ce qui est encore possible, que le monarque désire accorder les pouvoirs les plus illimités possibles, il demeurait nécessaire, dans l'hypothèse où des Anglais avaient déjà des droits acquis sur certaines découvertes, de réserver ces droits. Le 9 décembre 1502 , le roi rééditait la même commission au même groupe, avec quelques changements dans les noms, mais en des termes semblables, indiquant donc qu'il n'y avait eu aucune découverte entre-temps. Mais le 6 décembre 1503, un document concernant la pension à payer à des Portugais, bénéficiaires des permissions précédentes, suppose qu'à cette date il y a déjà eu découverte. Cette date de 1503 paraît être la plus sûre pour la redécouverte du Groenland, bien qu'une chronique postérieure date de 1502 l'arrivée en Angleterre d'indigènes "clothed in Beestes skynnes" et mangeurs de "raw fflessch". Ce dernier trait indique assez clairement des Esquimaux (Biggar, Les Précurseurs, 40-59, 70-92). Ainsi, les Portugais, qui semblent avoir 
Le document mérite l'attention. La première impression qu'il donne est que le découvreur anonyme a touché une côte nord-sud située à l'ouest et faisant face à l'Europe. Suivant cette impression, on est tenté de voir cette côte représentée sur la carte Oliveriana-Pesaro ${ }^{30}$, qui indique, à peu près sur la longitude $d u$ Groenland, une île rectangulaire dont l'un des longs côtés fait face à l'Europe et qui est appelée l'insula de Labrador. En y pensant bien, toutefois, la relation entre la description de Day et l'insula de Labrador n'est pas fondée. Dans la lettre, le cap nord de la découverte est un cap de la terre ferme, et non d'une île, et il est situé sur la latitude de Dursey Head, en Irlande, tandis que l'insula de Pesaro ne monte pas plus haut que La Rochelle, en France.

Selon John Day, le point le plus méridional de la découverte est le sud de l'île des Sept-Cités, qui est sur la latitude de la rivière de Bordeaux, c'est-à-dire de la Gironde. Tandis que le sud de l'insula de Labrador est à la hauteur de la Coruña en Espagne.

En outre, c'est une erreur de se représenter la côte de John Day comme orientée directement du sud au nord. Les distances

déjà aperçu le Groenland en 1500 (ibid., 65), y conduisirent les Anglais en 1503. Les premiers considéreront ce pays comme une découverte portugaise et l'appelleront Labrador; pour les seconds, il sera une découverte anglaise, désignée sous l'appellation de Newfoundland.

30 M. Theodore E. Layng, dans une étude faite en 1961 ("Charting the course to Canada", tiré à part d'un texte publié dans Actas do Congresso internacional de Historia dos Descobrimentos, II, Lisboa, 1961), est d'avis que cette carte a été faite d'après un prototype révélant une exploration de l'Amérique avant Cortereal. L'existence d'un tel prototype, en dehors de la carte La Cosa et des cartes consécutives à Cortereal, ne s'impose pas. Elle ne pourrait être établie que par une tradition où un tel prototype aurait exercé une influence certaine sur plusieurs cartes indépendantes les unes des autres. Et encore, il devrait être établi que ce prototype est plus ancien que les cartes plus haut mentionnées. Tel n'est pas le cas ici, la forme particulière des territoires nord-atlantiques, dans l'Oliveriana, pouvant être suffisamment expliquée par l'influence conjuguée de la carte La Cosa et d'informations portugaises. Le Groenland de l'Oliveriana, descendu de $60^{\circ}$ à $51^{\circ}$ de latitude, ne témoigne pas particulièrement d'une information originale et objective. Les cartes de la tradition Cortereal se rapprochent davantage du vrai et l'Oliveriana marque un recul, qui s'expliquerait par l'influence de la carte La Cosa. En principe, la méthode, en ce domaine, doit être de s'en tenir à ce que les cartes expriment prima facie et de réduire les conjectures au strict minimum. Autrement, il ne sera jamais possible de se mettre d'accord. 
ont un tout autre langage. La lettre laisse entendre que l'exploration a commencé au sud et s'est terminée au cap du nord, qui est à 1800 milles de l'Irlande. Ce cap est à quinze jours de navigation de la côte de la Bretagne française. Le voyage qu'il a fallu faire pour aller trouver le sud de la terre neuve a duré 35 jours. Les conditions de la navigation ont été à peu près idéales. A l'aller, le vent était est-nord-est; au retour, il soufflait en poupe. On a donc eu constamment vent arrière. Ainsi, les longueurs de l'aller et du retour donnent une idée approximative des distances du sud et du nord du nouveau continent par rapport à l'Europe. L'exploration, s'étant faite du sud au nord, à duré un mois. Si l'on met toutes ces données sur un dessin compris entre le $45^{\mathrm{e}}$ degré et 35 minutes de latitude nord, hauteur approximative de la Gironde, et le 51e degré et 30 minutes, hauteur approximative de Dursey Head, on se fait une représentation des proportions et de l'orientation des terres découvertes (fig. II).

Ce qui saute alors aux yeux est que cette représentation a exactement les mêmes proportions que la côte de la carte La Cosa. On sait que cette dernière côte a été inspirée par une source anglaise, du genre de la lettre de John Day. Comme dans la description de John Day, le cap le plus méridional de La Cosa, avoisiné par une grande île qui a son extrémité sud à la même hauteur, est sur le parallèle de la rivière de Bordeaux. Dans les deux documents, le cap le plus septentrional est sur le parallèle du sud de l'Irlande. L'exploration de la côte de la Cosa s'est faite du sud au nord, comme l'indique la direction des drapeaux; celle du découvreur de John Day, également. Le sud et le nord de la côte sont éloignés de l'Europe dans la même proportion que les longueurs données par John Day pour l'aller et le retour. La lettre de ce dernier ne donnait aucun nom de découvreur; la carte n'en donne pas non plus: mar descubierto por Ingles, mer découverte par un Anglais. Ces coïncidences rares et étonnantes, jointes à l'absence de toute divergence, me font conclure que l'auteur de la carte La Cosa a tracé cette côte d'après le dessin que John Day a expédié au grand Amiral. Car un tel dessin accompagnait la lettre: "la 
copia de la tierra que es fallada". Et le texte renvoie le lecteur à ce document, pour connaître les détails toponymiques: "Mais par ladite copie Votre Seigneurie aura l'intelligence de ce qu'elle veut savoir, parce que, dans la même copie, sont nommés les caps de la terre ferme et les îles; et ainsi elle verra du même coup le lieu qui fut aperçu en premier, car c'est au retour qu'on a trouvé la plus grande partie de la terre découverte ${ }^{31 . "}$ Ainsi, la toponymie de La Cosa ne peut guère avoir d'autre origine que ce même dessin. Le cartographe, toutefois, semble avoir pris le parti de repousser tous les noms sur la moitié est du dessin, probablement pour les rejeter en dehors de l'hémisphère espagnol ${ }^{32}$. Ainsi est disparu le nom de la grande île des Sept-Cités et le cabo descubierto ${ }^{33}$ est fort loin de cette île où l'exploration a débuté, à l'extrémité sud-ouest ${ }^{34}$.

Si l'on accepte de relier les deux documents, ils s'éclairent mutuellement d'une façon inespérée. La description de John Day acquiert une forme visuelle qui ne laisse plus de place à la discussion; la côte de La Cosa reçoit une explication qui balaie $d u$ coup un monceau d'interprétations et d'hypothèses sous lequel on l'avait enterrée pour la rendre vraisemblable. Les

31 “... pero por la dicha copia comprendra Vuestra Señoria lo que quiere saber, que en la misma copia estan nombrados los cabos de la tierra firme $\mathrm{y}$ las islas, y por alli asy mismo verà donde fue la primera vista, porque a la vuelta se fallo la mayor parte de la tierra que se descubrio" (Hispanic American Historical Review, XXXVI: [507]).

32 Le cartographe, selon une pratique qui se rencontre aussi chez ses collègues, a tassé la nomenclature sur la moitié orientale de la côte. La lettre de John Day indique que le nom de l'île des Sept-Cités se trouvait sur le dessin original. La manœuvre du cartographe lui a fait omettre cette indication. La raison pourrait avoir été de ne laisser aucune toponymie anglaise à l'ouest de la ligne de Tordesillas, c'est-à-dire en territoire espagnol. Une comparaison avec la côte du Vénézuela suggère fortement un tel motif.

${ }^{33}$ L'indication cabo descubierto n'est plus lisible aujourd'hui sur l'original. Nous faisons confiance à ceux qui l'ont lue les premiers, au dix-neuvième siècle.

34 Le cartographe a probablement réduit le dessin de John Day, tout en conservant les latitudes et les proportions. Le mille de John Day était sans doute le mille nautique, qui est le tiers d'une lieue. Celui de la carte La Cosa paraît être le mille romain, qui est le quart d'une lieue. Mesurée en milles nautiques, la découverte se serait étendue bien plus loin à l'ouest que les découvertes de l'Espagne et l'orgueil hispanique en aurait souffert. 
distances, les orientations, les traits du dessin prennent une netteté et une solidité difficilement évitables.

Pour porter un jugement sur ces deux sources qui n'en font qu'une seule, je pars de ce principe qu'un découvreur doit décrire les choses comme elles sont dans la réalité géographique. Je tiens compte des illusions d'optique possibles. Mais la substance de la description doit être vérifiable sur les lieux; si au contraire le découvreur affirme catégoriquement l'existence de ce qui n'existe pas, je le tiens pour un imposteur. Ce qui est affirmé, dans la lettre de John Day et sur la carte La Cosa, c'est que, entre les degrés de latitude nord quarante-cinq et demi et cinquante et un et demi, commence, à 1800 milles de l'Irlande, une côte est-ouest regardant généralement vers le sud et plus longue que deux mille cinq cents milles, où les fleuves coulent du nord au sud. Je dis qu'une telle côte n'existe pas et que, par conséquent, le découvreur qui a affirmé l'avoir explorée est un imposteur. Le faux est si manifeste que je n'ai pas besoin d'entrer dans les détails.

Je ne suis pas seul à porter un tel jugement sur cette prétendue découverte. Toute la critique du seizième siècle l'a fait unanimement, en négligeant le dessin de la carte La Cosa. C'est ici que la carte de Pesaro paraît spécialement instructive. Elle est une carte d'origine espagnole, selon la langue qui y est employée avec le latin ${ }^{35}$. L'influence de la côte La Cosa sur cette carte est nettement perceptible, d'abord à propos de la latitude du Groenland, descendu jusqu'à la hauteur du sud de l'Irlande, puis de la forme de Terre-Neuve, un dessin La Cosa énormément raccourci. Mais en dehors de là, le cartographe abandonne la source anglaise et y substitue les informations des sources portugaises : séparation du Groenland et de Terre-Neuve, dimension des côtes, dessin du Groenland et surtout nomenclature. La particularité la plus étonnante est que l'auteur a conservé l'orientation est-ouest de La Cosa à la côte de Terre-

35 Par exemple, on ne peut s'expliquer autrement que grâce à un intermédiaire espagnol cette transcription d'un nom portugais sur une carte italienne: riuo de los bacalaos. L'article n'est ni portugais ni italien, mais espagnol. 
Neuve. Mais la toponymie est portugaise, celle de la source anglaise ayant été abandonnée. L'ordre des noms est étrange. Cela ne s'explique qu'en partie par l'orientation de la côte, car ils sont disposés de l'ouest à l'est, alors qu'il eût été plus naturel de les aligner de l'est à l'ouest. Une considération supplémentaire a dû mouvoir le cartographe. On la trouve, semble-t-il, dans le cavo del Marco, qui désigne, en portugais, la limite de Tordesillas. Comme le territoire portugais doit se trouver à l'est de cette dernière ligne, le Marco a été placé à l'ouest et les autres noms s'échelonnent vers l'est. Dans la réalité, le Marco des Portugais était situé au nord et les autres noms venaient plus au sud. Certains de ces noms ont une solidité historique à toute épreuve, Bonaventura (Bonavista), Bacalhao (Baccalieu), de la Spera (Spear), parce que les marins les employaient dès ce temps-là sur les lieux où on les voit encore aujourd'hui. La conséquence à tirer de ces faits est la suivante: l'auteur de la Pesaro a jugé les sources portugaises préférables à l'ancienne source anglaise de La Cosa, qu'il a sacrifiée à peu près entièrement. Mais la forme de Terre-Neuve et la latitude du Groenland, conservées de La Cosa, montrent à quel point étaient encore déficientes les connaissances des Espagnols sur les découvertes portugaises du nord. Il est probable, toutefois, que l'auteur savait quelque chose du lien qui unissait l'Angleterre au Labrador. Je crois que c'est ce qu'il a voulu indiquer par cette insula de Labrador, qui n'a aucune réalité géographique.

La carte La Cosa dépend du dessin de John Day, mais a-t-elle quelque parenté avec la carte que Pedro de Ayala n'a pas envoyée, le 25 juillet 1498 ? Ce dessin de Day serait parvenu en Espagne dès l'automne de 1497, selon les conclusions de M. Vigneras. J'y vois une difficulté que je n'ai pas signalée plus haut. Je vous prie de bien lire la phrase de Ayala qui se rapporte à ce sujet: "Parce que Vos Altesses doivent déjà avoir avis de tout cela et même aussi de la carte ou mappemonde que celui-là a faite, je ne l'envoie pas maintenant, parce que je l'ai ici, et à mon avis bien fausse, pour donner à entendre 
que ce ne sont pas les dites îles ${ }^{36}$." Si le dessin de John Day est identique à celui de la mappemonde de Jean Cabot, je ne vois vraiment pas de quoi se plaint l'ambassadeur. Car la côte La Cosa et la lettre de Day montrent, sans contestation possible, la découverte dans le domaine de l'Espagne, sans que ce fait soit camouflé le moins du monde; bien au contraire. Je ne vois qu'une conclusion à tirer de cela: le dessin de John Day est autre chose que la carte faite par Jean Cabot. Et il s'ensuit qu'il n'est pas de 1497.

Mais, dira-t-on, la carte de La Cosa elle-même est de 1500 et, avant 1500, on ne voit pas quel autre que Jean Cabot aurait pu rapporter des informations sur un monde nouveau situé dans le nord de l'Atlantique. Je sais qu'une inscription de la carte en fait foi, sous l'image de saint Christophe: "Juan de La Cosa la fizo en el puerto de Santa Maria en año de 1500"; Jean de La Cosa l'a faite au port de Santa-Maria en l'année 1500. J'admets bien que, si la carte était vraiment de cette année, l'attribution de la découverte à Jean Cabot gagnerait un appui considérable. Dans ce cas, le millésime de 1497 deviendrait fort vraisemblable pour la lettre. Mais alors, il faudrait dire que Jean Cabot a donné deux versions simultanées de son voyage, deux versions opposées et inconcilíables: celle de Soncino et celle de John Day. Ce n'est pas très vraisemblable. Sébastien Cabot, certes, s'est montré capable de palinodies pareilles. Mais au moins, il les espaçait dans le temps et n'en soutenait pas deux à la fois.

En fait, la carte qui est aujourd'hui attribuée à La Cosa n'est pas de lui et elle est postérieure à 1500. Sans doute Jean de La Cosa est-il l'auteur d'un original dessiné en 1500. Mais notre exemplaire est une copie, qui n'est certainement pas de La Cosa lui-même. Ce qui le prouve, c'est l'abondance des corruptions de la nomenclature, spécialement sur la côte du Vénézuela, explorée par le cartographe lui-même en 1499-1500.

36 "Porque creo Vuestras Altezas ia tendran aviso de todo esto y ansimismo al (sic) carta o napamundi (sic) que este ha fecho. Io no le enbio aora, que aqui le ai, y a mi ver bien falso por dar a entender, no son de las islas dichas" (Biggar, Les Précurseurs, 27-28). 
Harrisse s'est plu à faire de longues listes de ces noms rendus inintelligibles par les copistes et que La Cosa n'avait pu écrire de la sorte ${ }^{37}$. Un seul copiste ne suffit même pas à rendre compte de ces fautes; il faut que plusieurs aient passé successivement sur les mêmes noms ${ }^{38}$. C'est aussi La Cosa qui a exploré le golfe de Darien, en Colombie, durant les années 1501-1502. Ce golfe est inscrit en traits généraux, mais incontestables, sur notre carte. La Cosa, qui en connaissait très bien la côte, n'est certainement pas celui qui l'a dessinée sur notre carte. Il y aurait d'ailleurs mis la nomenclature, comme il l'a fait pour le Vénézuela.

George E. Nunn a savamment discuté cette question de la date de notre carte ${ }^{39}$. On ne reprendra pas ici son argumentation. Mais le principal raisonnement semble être celui-ci. La Cosa a conçu sa carte comme une mappemonde, c'est-à-dire comme une représentation complète de la terre, à un moment où il croyait, avec Colomb, avoir approché le littoral oriental de l'Asie. Or la carte actuelle démontre qu'elle a été achevée en un temps où l'on avait déjà reconnu que la côte américaine ne correspondait pas à celle de l'Asie, où il deviendrait, par conséquent, nécessaire de chercher un passage au-delà du nouveau monde pour atteindre le but primitif des découvertes.

\footnotetext{
${ }^{37}$ Henry Harrisse, The Discovery of North America, (Paris-London, 1892), 414-415.

38 Nous prendrons un exemple sur la côte du Vénézuela, au dernier point à l'ouest du territoire visité par La Cosa en 1499, c'est-à-dire à la Sierra Nevada de Santa Marta. Ce nom de Santa Marta remonte jusqu'aux premiers temps de l'occupation espagnole et il n'y a guère de doute qu'il n'ait été donné durant le voyage de découverte. L'exploration de cette année-là a fini à cet endroit. Sur la carte de La Cosa on lit difficilement un nom que Harrisse a rendu par montes de Santa Eufemia. Sur la meilleure reproduction existante (Mapas Españoles de America (Madrid, 1951), pl. I et II), on voit $m$ de $s$ (eu?) femja. La première syllabe du nom est peu lisible. Le $f$ pourrait être un $s$. De toute façon, le nom de Santa Eufemia n'est pas connu dans la région. Nous voyons l'évolution de cette inscription comme suit: sur l'original, montes de $\boldsymbol{s}^{a} \mathrm{~m}^{\text {ta }}$; un premier copiste l'a transformée en montes de $s^{a}$ mja (montes de Santa Maria); le troisième a lu difficilement le mot montes et l'a altéré en $m$ de $s$; une fois induit en erreur, il a produit cet ensemble qui n'est guère lisible: $m$ de $s$ (de?) femja. Il semble, en tout cas, qu'il ait fallu plus qu'un copiste pour arriver à ce résultat.

39 George E. Nunn, The Mappemonde of Juan de La Cosa. A critical Investigation of its Date (Jenkintown, 1934).
} 
Aussi Nunn est-il enclin à dater notre carte La Cosa des années 1508 à 1510. De toute façon, elle ne peut pas être antérieure à 1504 .

Vous voyez d'ici les conséquences pour l'attribution de cette découverte. Il n'est plus nécessaire de rattacher la lettre de John Day et la côte La Cosa à l'expédition de Jean Cabot ${ }^{40}$. En fait, puisque l'exploration décrite par elle est fausse, comme il a été reconnu, il est naturel de penser que le faux découvreur s'est inspiré de découvertes déjà faites. Il y en a des indices. Les 1800 milles sont la distance entre l'Angleterre et le Groenland par la route de l'Islande. L'allusion aux marchands de Bristol situe le lettre après 1503. L'équerre que fait la côte La Cosa au quart de sa course vers l'est ne rappelle-t-elle pas beaucoup certaines représentations de Terre-Neuve qui commençaient à circuler dans les éditions de Ptolémée de 1506 et de 1508 ? Souvenons-nous que Sébastien Cabot, en 1516, prétendra avoir longé une côte est-ouest en direction de la Chine. Tout cela, ajouté à la psychologie que révèle la lettre de John Day, si semblables à celle des récits du même Sébastien, m'incline fortement à attribuer ce chef-d'œuvre au fils de Jean Cabot. N'est-ce pas par de tels moyens qu'il a réussi à se faire appeler en Espagne en 1512 ? Au moins, je vois là une possibilité.

Pour revenir au terrain plus solide des faits, replaçons le problème de Jean Cabot dans le contexte de l'histoire des découvertes. Si le Vénitien, en 1497, avait découvert, comme le disent les sources analysées plus haut, un pays fertile, offrant la douceur de son climat, la richesse de sa forêt et de ses

40 Signalons ici l'une des plus récentes thèses sur le trajet de Cabot, étude remarquable pour les connaissances maritimes qui y sont déployées. Elle est de Melvin H. Jackson ("The Labrador Landfall of John Cabot, 1497", The Canadian Historical Review, juin 1963). Elle est typique du genre d'études qui se font sur ce sujet, parties d'hypothèses ingénieuses, mais indifférentes au contenu des sources historiques. Cet auteur rejette la carte de La Cosa comme trop douteuse, mais il prétend s'appuyer sur la lettre de John Day. Or il déplace sans scrupules les deux latitudes qui y sont données; il prend pour le point d'atterrage ce qui est pour John Day le dernier cap aperçu; il place sur une île le point de départ, que John Day situait sur le continent; et il ne tient aucun compte du rapport des longueurs entre l'aller et le retour. De cette façon, on montre bien ce qu'aurait pu être le voyage de Jean Cabot, mais non ce qu'il a été. 
pêcheries, avec la possibilité d'explorations plus étendues et plus profitables encore, l'heure de l'Angleterre n'aurait-elle pas été avancée d'un siècle ? Henri VII n'a pas manqué d'intérêt pour les récits de Cabot, en 1497. Pourtant, à partir de 1498, c'est le silence parfait sur le nom du découvreur, silence que Sébastien lui-même n'ose pas rompre avant $1544^{41}$. On ne trouve en Angleterre aucun mouvement semblable à celui qui a été déclenché en Espagne par la découverte de Colomb. Toujours, les découvertes officielles ont provoqué la création ou le renouveau d'une cartographie américaine chez le peuple découvreur. Or l'Angleterre n'a eu aucune tradition cartographique sur l'Amérique avant le dernier quart du siècle et ses explorateurs ne commencent à s'intéresser tout de bon au nouveau continent que vers 1580. Cette indifférence s'explique à l'égard du Groenland, dont le climat interdit l'exploitation; mais elle serait inexplicable à l'endroit de l'Amérique du Nord. Henri VII, qui avait assuré à Cabot et à ses héritiers le gouvernement et la jouissance économique de leurs découvertes ${ }^{42}$, ne réservera aucun droit acquis à cette famille, dans les lettres patentes accordées aux marchands de Bristol, en 1501 et 1502 . Si elle avait eu de tels droits, le monarque ne pouvait les oublier quatre ou cinq ans après les avoir accordés. On ne verra aucun pêcheur anglais à Terre-Neuve durant la première moitié du seizième siècle ${ }^{43}$. Enfin, les Cabot n'ont pas fait la moindre contribution au développement des connaissances européennes sur l'Amérique du Nord ${ }^{44}$.

41 La première fois que Sébastien donne à son père une participation aux découvertes que lui-même prétend avoir faites, c'est sur sa carte de 1544.

42 Biggar, Les Précurseurs, 6-10.

43 On voit deux navires anglais dans les eaux de Terre-Neuve durant la première moitié du seizième siècle, l'un en 1527 (Biggar, Les Précurseurs, 165-177), l'autre en 1536 (Biggar, The Voyages of Jacques Cartier, Ottawa, 1934, 273-277). Les deux sont montés par des explorateurs, non des pêcheurs. Le premier est égaré; le second est venu visiter le littoral. Mais il est évident que ces Anglais n'ont aucune expérience de ces parages. Pour le reste, on ne voit jamais de pêcheurs anglais dans les environs, bien que les Portugais, les Français et les Espagnols y soient signalés.

${ }^{44} \mathrm{La}$ carte de Sébastien Cabot, de 1544, ne rappelle en rien la carte de La Cosa. Elle s'insère simplement dans la cartographie espagnole contemporaine et elle s'inspire, pour l'Amérique du Nord, de cartes françaises comme celle de Desliens. 
Si Jean Cabot avait, en 1497, découvert l'Amérique du Nord, nul n'aurait été mieux placé pour le savoir que son fils, Sébastien. Celui-ci ne peut manquer $1^{\circ}$ d'être certain du fait, $2^{\circ}$ de savoir au juste ce que son père a vu ${ }^{45}$. Or quand on est certain d'un fait, on en donne toujours le même témoignage, au moins en substance. C'est un signe de la véracité. Il était d'autant plus facile à Sébastien de faire savoir à l'Europe ce qu'il tenait de son père que, jusqu'à 1520 et même après, on était très mal informé sur l'Amérique du Nord en dehors du Portugal; même les Portugais ne connaissaient qu'une petite partie du nouveau monde septentrional. Les hésitations de Waldseemüller, en 1507, et l'inexactitude de la carte OliverianaPesaro, encore postérieure, sont des preuves de l'ignorance générale. Mais Sébastien n'a fait que raconter des histoires idiotes, incroyables et bientôt démenties par les explorateurs; et il change son récit à chaque nouveau développement de la cartographie nord-américaine ${ }^{46}$. Toutes ces circonstances forment une convergence qui n'est pas sans signification. Et cette signification, c'est qu'il n'y eut pas de découverte de l'Amérique du Nord par l'Angleterre en 1497.

En effet, il est certain que les Anglais n'ont pas tenu Jean Cabot pour un découvreur, après 1498. Non seulement ils ne parlent pas de lui, mais John Rastell, en 1519, témoigne impli-

45 Un trait constant des récits des Cabot est le général et le vague, alors qu'une exploration se prouve par les indications concrètes et précises qu'on en rapporte.

46 La manie de Sébastien est de se donner pour le découvreur de la route de la Chine. Chaque fois qu'il soupçonne un nouveau passage, il y met son drapeau, comme étant celui qui l'a vu le premier. Ainsi longe-t-il à partir du cap de Raze une côte qui se prolonge à l'ouest jusqu'à la Chine, selon le récit de D'Anghiera. Puis il prétend être monté jusqu'au cercle polaire, à l'entrée d'un fretum indiqué sur quelques cartes comme allant en Chine. Une autre fois, il longe l'Amérique du Nord depuis le $56^{\mathrm{e}}$ degré jusqu'à la Floride: cette fois, il ne découvre aucun passage. A l'entrée du détroit de Cabot, il ouvre un autre passage vers l'ouest sur sa carte de 1544. Enfin, avant de mourir, il proclamera encore qu'il a été le premier à ouvrir la route de la mer de Barents vers la Chine. A remarquer, il s'agit toujours du même voyage de découverte, excepté pour le dernier. Mais les récits de Sébastien arrivent toujours après que d'autres ont fait les découvertes, quand celles-ci sont authentiques. 
citement contre lui, dans son poème, The new Interlude ${ }^{47}$. Ce poète ne semble pas connaître les exploits de Christophe Colomb. Mais il a eu connaissance des récits de Vespucci, publiés en 1507 par Waldseemüller, puisqu'il donne le nom d'Amérique au nouveau continent. Il attribue, selon Waldseemüller, la première découverte des New founde landes à Vespucci, mais il dit aussi que Henri VII a eu la première initiative d'y envoyer ses découvreurs. Quoi qu'il en soit de l'accord entre ces deux propos, le poète affirme cependant:

This See is called the great Occyan.

So great it is that never man

Coude tell it sith the worlde began

Tyll nowe within this $X X$ yere

Westwarde be founde new landes

That we never harde tell of before this

By wrytings nor other meanys.

Les démarches de Henri VII sont peut-être relatives aux expéditions tentées à partir de Bristol, y compris celles de Cabot. Mais les vingt dernières années, pendant lesquelles on a commencé à entendre parler de la nouvelle terre, ne remontent pas plus haut que 1499. En cette dernière année, Vespucci, d'après les narrations publiées par Waldseemüller, rapportait en Europe les premières nouvelles de ses découvertes. Et il y aura, trois ou quatre ans plus tard, la redécouverte du Groenland par une expédition anglo-portugaise. Mais le voyage de Jean Cabot, en 1497, est de deux ans antérieur à cette période de vingt ans. Ainsi, John Rastell ne tient pas le Vénitien pour un découvreur qui ait fait connaître le nouveau continent à l'Europe.

En 1555, Richard Eden publie en traduction diverses œuvres racontant la découverte de l'Amérique et en faisant la description ${ }^{48}$. Il relève avec complaisance les mentions de Sébastien Cabot, qui est alors revenu vivre en Angleterre, mais le nom

47 James A. Williamson, The Voyages of the Cabot and the English Discovery of North America under Henry VII and Henry VIII (London, 1929), 89.

48 Richard Eden, The Decades of the newe Worlde or West India..., (Londini, In ædibus Guilhelmi Powell, Anno 1555). 
de Jean Cabot n'apparaît pas dans son ouvrage, non plus que les voyages de 1497 et de 1498. Vers 1578, John Dee revendiquait l'Amérique du Nord pour la couronne d'Angleterre. Antérieurement à l'expédition de Frobisher, de 1576, le titre le plus récent invoqué par lui est le suivant: "Circa An. 1497, Sebastian Caboto, sent by King Henry the seventh, did discover from Newfound Land so far along and abowt the Coaste nexte to Labrador tyll he came to the Latitude of $671 / 2$. And styll fownd the Seas open before him ${ }^{49}$." Il n'est pas encore question de Jean Cabot chez les écrivains anglais. Et le voyage auquel Dee se rapporte est la plus invraisemblable des inventions de Sébastien, telle que racontée dans la préface du troisième volume de Ramusio, publié en 1554 .

Hakluyt a été le propagandiste de la légende de Jean Cabot en Angleterre. Il avait découvert les premières lettres patentes octroyées à notre héros par Henri VII et il les publia en 1582. Mais ce sont les voyages de Sébastien qu'il va attribuer au père, car il ne semble avoir connu aucun document authentique sur l'épisode auquel Jean a été mêlé. Ainsi, toute la preuve d'un voyage de Jean Cabot en Amérique du Nord a reposé, jusqu'au dix-neuvième siècle, sur le témoignage de Sébastien et sur la confusion qu'a faite Hakluyt. Or on sait que Sébastien, sur ce sujet précis, ne mérite aucune confiance ${ }^{50}$. Lorsqu'on découvrit

49 Williamson, The Voyages of the Cabots..., [23].

50 Malgré qu'on en ait, on trouve toujours Sébastien sur sa route, lorsqu'on traite de cette question. M. Layng, dans l'étude déjà citée ( $c f$. supra, n. 30), fait grand état du témoignage de Santa-Cruz (Biggar, Les Précurseurs, 183-185) pour établir que Jean Cabot est passé par le Groenland en 1497. Ce témoignage de Santa-Cruz est suspect pour beaucoup de raisons, entre autres à cause de sa date reculée, 1541. Il l'est encore à cause de l'erreur sur le nom de Cabot. Santa-Cruz le nomme Antonio. Au moins une partie de ses renseignements viennent de la Schondia de Ziegler. Or c'est Ziegler qui a inventé le nom d'Antoninus pour Cabot. Et cet Antoninus, c'est Sébastien, auquel se rapporte l'épisode évoqué par Ziegler et repris par Santa-Cruz, tous deux dépendants du témoignage de PierreMartyr d'Anghiera. Santa-Cruz fait d'Antoninus, devenu pour lui Antonio, le père de Sébastien. Son autorité, sur ce point, est on ne peut plus suspecte. Et Richard Eden ne s'y est pas trompé, puisqu'il a restitué Sébastien à la place d'Antoninus en traduisant Ziegler. Les confusions faites par SantaCruz, sur ce point et sur plusieurs autres, ajoutées à sa date tardive, sont loin de fournir une base solide à une thèse qui est déjà en désaccord avec les aocuments contemporains de Jean Cabot. 
de nouveaux documents, au siècle dernier, on les interpréta comme des confirmations de l'opinion courante, alors qu'en réalité il y avait entier désaccord, et sur la substance et sur les détails.

Telles sont mes raisons de ne pas accepter comme un fait historiquement établi que Jean Cabot a découvert l'Amérique en 1497. Ce fait doit d'abord être démontré, avant toute recherche du lieu où Cabot a débarqué. Je ne suis pas du tout fier d'être sur ce point en opposition avec l'ensemble des historiens nord-américains. Si jamais on établit valablement le contraire de ce que j'ai soutenu, je serai heureux de me rendre à de bons arguments et je ne m'obstinerai pas. Mais je supplie que l'on commence par une saine critique des sources, que l'on produise des raisons solides, non des échafaudages de postulats et d'hypothèses comme il $\mathrm{y}$ en a beaucoup trop dans le traitement de cette question. La solution de ce problème me paraît de très grande importance, car l'histoire de la découverte de notre pays a été entièrement faussée et embrouillée par ce que j'appellerais volontiers la légende des Cabot.

LUCIEN CAMPEAU, s.j.

N.D.R. La liste des thèses présentées à l'Institut d'Histoire (1947-1965) de l'Université de Montréal paraîtra dans notre prochaine livraison. 